\title{
Teknokultura
}

ISSNe: $1549-2230$

http://dx.doi.org/10.5209/TEKN.57192

\section{La narración colaborativa en @Elhombredetweed. Un análisis pragmático para el estudio de la literatura digital}

\author{
Luis Felipe González Gutiérrez ${ }^{1}$
}

Recibido: 29 de octubre de 2017 / Revisado: 6 de diciembre de 2017 / Aceptado: 26 de diciembre de 2017 Open peer reviews

Resumen. Se discute en este artículo los resultados del análisis de la cuenta de Twitter @ Elhombredetweed, a partir de los aportes de la literatura digital, específicamente la poesía digital, el construccionismo social y la psicología cultural. A partir de estos resultados se reflexiona sobre el impacto de estas escrituras colaborativas en los estudios actuales sobre las humanidades digitales, aplicadas al campo de la literatura electrónica. La metodología del estudio es de corte cualitativo, específicamente orientada a la investigación poética. Para la descripción y análisis de los tuits se trabajó con el software de análisis de redes sociales Netlytic. Los resultados indican dos historias que aparecen de manera episódica y secuencial en la cuenta de Twitter: La historia-objeto del escritor mexicano Mauricio Montiel (autor de la cuenta) y la serie de historias construidas por los seguidores de @ Elhombredetweed, lo que configura un ejemplo de narrativa transmedia y muestra el impacto de las redes sociales en la construcción colectiva de historias y la formación de subjetividades digitales, a través del uso de las TIC como potencializadoras de una realidad online.

Palabras clave: construccionismo social; humanidades digitales; investigación poética; literatura electrónica; Twitter.

\section{[en] The collaborative writing in @Elhombredetweed. A pragmatic analysis for the digital literature studies}

\begin{abstract}
This article discusses the preliminary results of the analysis of the Twitter account (a)Elhombredetweed account, from the contributions of digital literature, specifically on digital poetry studies, social construccionism and cultural psychology. From these results indicates the importance of the collaborative writing in the actual studies in the digital humanities, especially in the digital literature field. The methodology of the study is centered in poetic research, like a qualitative approach. For the tweets analysis we worked with the social network analysis software Netlytic. The results indicate two stories in the account: the literary story-object of the Mexican writer Mauricio Montiel (account author) and the series of projects and narrative sequences of the followers to @Elhombredetweed, which constitutes an example of transmedia narrative and shows the impact of social networks in the collective construction of stories and the formation of digital subjectivities, through the use of ICT as a potential for an online reality.
\end{abstract}

Keywords: digital humanities; electronic literature; poetic inquiry; social construccionism; Twitter.

Sumario. 1. Introducción. 2. Método. 3. Población. 4. Instrumentos. 5. Procedimiento. 6. Resultados. 7. Discusión de resultados. 8. Conclusiones. 9. Referencias.

Cómo citar: González Gutiérrez, L.F. "La narración colaborativa en @Elhombredetweed. Un análisis pragmático para el estudio de la literatura digital”: Teknokultura, vol. 15 (1) 2018, pp. 5-22.

1 Universidad Santo Tomás (Colombia)

E-mail: luisgonzalez@usantotomas.edu.co 


\section{Introducción}

Las aproximaciones narrativas en los diferentes campos de las ciencias sociales, sobre todo en la era digital, se han visto expandidas a una serie de autonarraciones y formas experimentales de escritura que han cumplido, con eficacia absoluta, la idea ya desarrollada por Gergen en sus trabajos clásicos sobre la construcción social de la realidad y los principios construccionistas sobre el yo relacional y el concepto de significado, en tanto una posesión comunitaria y cultural (Gergen y Gergen, 2011; Gergen, 2016, 2006, 1998). Si bien las contribuciones del construccionismo social sobre la construcción social del conocimiento se han dado en un momento histórico en el que las tecnologías no estaban desarrolladas como en el momento presente, la expansión de lo digital en nuestra sociedad es un hecho que ha permitido comprender que el yo relacional, la mediación de las relaciones interpersonales y el sentido de construcción social de la realidad se expanden y potencializan por la cibercultura, la inteligencia colectiva (Levy, 2007, 2004) y el desarrollo teórico y práctico alrededor de las humanidades digitales, como apuesta para comprender los retos de la postmodernidad, específicamente centrados en la relación del hombre con las máquinas y los sistemas de información y comunicación.

A su vez, en el campo de las humanidades digitales, el interés por comprender la relación y uso de las tecnologías en el plano de los intercambios narrativos, es siempre un reto de abarcar en algunas líneas. Sin embargo, a modo de descripción sucinta, se esbozan algunos referentes teóricos que relacionan la propuesta de las humanidades digitales en la comprensión de fenómenos actuales en la literatura digital como lo son el digital storytelling, el digital storycicle, la escritura en colaboración, entre otros fenómenos creativos.

Es un hecho que el entorno de múltiples plataformas digitales, con sus correspondientes intercambios narrativos, genera un potencial de participación en personas jóvenes especialmente (Clark, Couldry, MacDonald y Stephansen, 2015). Asimismo, diversas experiencias alrededor del uso de estrategias digitales en literatura potencian, entre otros aspectos, procesos de aprendizaje y apropiación de la literatura en adultos jóvenes (Radovanović, Hogan y Lalić, 2015); integración de saberes disciplinares, tales como la relación entre el estudio de los negocios con las artes liberales (Nesteruk, 2015) y maneras específicas de reconocimiento mutuo surgidas del intercambio narrativo (Couldry et al., 2015). Estos ejemplos describen con acierto la importancia de apropiar saberes, conocimientos y aprendizajes mediados en entornos digitales. Otro aspecto a considerar del uso de lo digital en el campo de la literatura se da a partir de la experiencia de las conexiones entre usuarios, las cuales potencian el trabajo creativo y alimentan e integran experiencias de aprendizaje, específicamente vinculadas al uso de las redes sociales como estrategia de aprendizaje (Quan-Hasse, Suarez y Brown, 2015) o centradas en el entretenimiento y la relación hombre-máquina (Kateros, Georgiou, Papaefthymiou, Papagiannakis, y Tsioumas, 2015).

Sin duda, como afirma Tsatsou (2017), resulta necesario establecer una serie de entrenamientos sobre las significaciones frente a lo que es la literatura digital, así como identificar todos los recursos que esta forma de creación tiene a su haber para ser desarrollada en el campo de los estudios literarios digitales (Valls-Russell, 2016). En resumidas cuentas, la cada vez más cercana relación entre la literatura 
digital y las alternativas que ofrece el mundo de las tecnologías de la información y la comunicación contribuye al estudio de las humanidades digitales en tres aspectos. El primero, como lo sostiene Deegan (2014) a propósito de los retos de las humanidades digitales, está en explorar la herencia cultural y comprender la condición humana, ya que el hecho mismo de la nueva condición del 'ser digital' transforma el escenario de las actuales humanidades. Un segundo aspecto tiene que ver con la cada vez más creciente interrelación del big data en la comprensión de las ciencias sociales. Como lo sostiene Wagner-Pacifici, Mohr y Breiger (2015), algunos temas a ser considerados como importantes en la interrelación del Big Data en ciencias sociales, se relacionan con la naturaleza de las descripciones que se realizan actualmente, las construcciones categóricas sobre individuo, entidades y agentes sociales; y la determinación de causalidad, entre otros aspectos teóricos cruciales. Un tercer aspecto, como lo sostiene Kitchin (2014), son las consecuencias epistemológicas a las que se ven abocadas las humanidades digitales, dado que se deben crear nuevas formas de sentido para comprender la cultura, la historia, la economía. Insiste, incluso, que las maneras como se investiga deben cambiar, en la medida que la mayoría de las metodologías de investigación (tanto cualitativas como cuantitativas) surgieron en el marco de la modernidad.

En el campo de los estudios sobre la era digital y las tecnologías de la información y la comunicación, importantes contribuciones se han realizado frente a la integración/complemento de las narrativas online y offline, referida a la construcción de la identidad en el mundo digital y el mundo análogo (Elwell, 2014), además de los relevantes aportes del concepto de transmedia storytelling (Jenkins, 2010, 2008, 2004; Jenkins y Deuze, 2004), sin olvidar los desarrollos teóricos y experienciales de la ecología de los medios, en su interacción con aspectos de la evolución y complejidad en el uso de los medios digitales, especialmente dirigidos a aspectos de la vida cotidiana (Scolari, 2013a, 2013b, 2013c).

Se puede afirmar que el desarrollo progresivo y sin pausa del mundo digital, que sin duda ha complejizado el mundo de las relaciones interpersonales y los procesos de comunicación, ha creado las condiciones para la reflexión teórica y metodológica sobre aspectos del mundo digital que han contribuido a comprender la identidad, no centrada en términos de un asunto privado e individual, sino de un sentido de colaboración, construcción colectiva y formación de identidades en red, integradas, al mismo tiempo vinculadas con experiencias que construyen modos alternos de subjetividad, ligadas a la experiencia relacional y compleja de dichas identidades, cada vez más diversas e insertadas en la actividad electrónica y análoga.

Dentro de los campos de reflexión teórica sobre estos temas en cuestión se consideran, entre otras, la construcción de la identidad en la construcción de memes en Internet (Gal, Shifman y Kampf, 2016); el desarrollo de la identidad de hombres adultos a través de los juegos en línea (Doh y Whang, 2014); la reflexión teórica por la consecución de modelos para comprender la identidad virtual en mundos virtuales (Nagy y Koles, 2014); la identificación de prácticas de identidad y privacidad en la comunicación de la vida digital diaria (Wessels, 2012); la conformación de las identidades personales online (Floridi, 2011); la introducción (y relectura) de los estudios de Goffman sobre la presentación del self en el mundo online (Bullingham y Vasconcelos, 2013) e, inclusive, aproximaciones a la construcción discursiva de la identidad de género en la social media (Cook y Hasmath, 2014). 
Ante este panorama, la literatura ha tenido también una transformación creciente sobre lo que se entiende por la creación literaria en medios digitales. El proceso de la escritura creativa, vinculada a las tecnologías de la información y la comunicación, se ha convertido en un género en sí mismo, por decirlo de alguna forma, lo que ha expandido los estudios de la literatura más allá de las fronteras del papel y el mundo análogo. En este sentido, Kozak (2015) define la literatura digital como: “...un tipo de práctica literaria que toma como base misma para su producción y recepción los recursos habilitados por la informática, desde la materialidad del hardware hasta la programación del software" (p. 93). Chico (2007) afirma que una teoría literaria en la era digital, basa su análisis en una propiedad significativa de la producción digital. La posibilidad de descomponer la obra en pequeños fragmentos de texto o hipertextos tienen dos características relevantes frente al acceso a esta literatura emergente: la posibilidad de una lectura no secuencial (diferente a la inducida en el texto análogo) y las múltiples posibilidades de lectura, que otorgan un poder especial y particular al lectoautor, palabra clásica introducida por Landow (1995, 1997). Para Chico (2007):

...el hipertexto, al presentar una red de textos o nodos que el lector puede recorrer libremente en todos sus sentidos, libera a éste de la secuencialidad cerrada y limitadora de la escritura tradicional, libertad del lector que muy a menudo se correlaciona con la suplantación —y, en los casos más extremos, negación- del autor como categoría constructora o creadora de la obra de arte verbal (p. 799).

Es de este modo que el creciente número de trabajos sobre ciberliteratura (o literatura digital) describe las infinitas posibilidades de construcción de textualidades a través del weblog, por ejemplo, (Cravero, 2011) o de la creciente participación del lector como parte del proyecto creativo, lo que ha dado impulso a una serie de experimentos ciberliterarios en los que se retoman las tradicionales técnicas surrealistas (como el cadáver exquisito), pero amplificadas en el plano de las plataformas web y las redes sociales, además de incluir aspectos relacionados al estudio sistemático de esta literatura (Romero, 2011; Begoña, 2012). Además de esto, organizaciones como la Electronic Literature Organization (ELO) han destacado una serie de contribuciones teóricas sobre aspectos cruciales en la relación de las humanidades digitales, como lo son las 'Humanities Computing' (Simanowski y Gattass, 2017); las narrativas improvisadas conectadas en red - Networked Improv Narratives o Netprov(Wittig, 2015); el interés creciente por las formas literarias menores (Szilak, 2015) o las reflexiones sobre obras específicas de la literatura, como es el caso del estudio sobre la obra Holes, de Graham Allen (Karhio, 2017).

En el campo de la poesía, como lo afirma Vilariño, una de los grandes aportes de la relación de lo digital en la poesía es una apuesta por romper con los determinismos del lenguaje en el mundo análogo. Para la autora: "Las obras digitales, y en especial las poéticas, combinan distintos sistemas semióticos, provocan una contaminación entre ellos y ponen en paralelo lo lingüístico, con lo visual y lo fónico" (Vilariño, 2013, p. 222). Esto ha permitido la creación de obras performativas, visuales, tridimensionales y al mismo tiempo interactivas, que traen al presente de lo digital, muchas de las formas modernas poéticas experimentales como la poesía concreta y los clásicos poemas-objeto del surrealismo. Esto sin duda ha tenido consecuencias en las 
formas de leer por poético, lo que lleva a importantes contribuciones en los procesos de creación y en sus aplicaciones en el campo de la educación (Neves, 2010).

Una de estas formas de escritura de poesía digital se puede expresar a través de Twitter. El uso de esta red social se convierte en un recurso importante en la comprensión del uso del big data para el empleo de análisis de las ciencias sociales a partir de la social media (Felt, 2016) o como un recurso que se puede incorporar en el campo de la enseñanza escolar (Quan-Hasse, Martin y McCay-Peet, 2015). E1 uso específico de Twitter como recurso literario digital, tiene una gran influencia dado que la restricción a 140 caracteres, permite pensar cada tuit como producto individual de creación (verso autónomo) que puede ser compartido, respondido y, en consecuencias, posibilita nuevos intercambios en los usuarios.

Para Zambrano (2015), la poesía que se produce mediante Twitter actúa como la minificción literaria en el sentido de la brevedad de los textos, al ser condensados, textos breves que integran lo poético y lo narrativo. Según el autor, este tipo de literatura producida en clave de Twitter se le denomina tuiteratura narrativa. Ante esta perspectiva, la utilidad de esta estrategia de escritura, de acuerdo a los principios de la psicología cultural (Cole, 1999; Esteban y Ratner, 2010; Glaveanu, 2010), resulta significativa en la medida que un artefacto social pasa de ser una actividad más en el plano de la creación a un recurso de reconstrucción de la identidad, ampliada por la creación y la interacción con otros usuarios de una red. En consecuencia, es de interés de este artículo comprender el uso colaborativo de la cuenta de Twitter @Elhombredetweed, como una estrategia de construcción de la subjetividad creativa en lo usuarios y del autor del proyecto literario, vinculado a esta red social.

\section{Método}

Se abordó esta investigación desde una perspectiva cualitativa (IC), centrada de forma específica en la investigación poética. Gracias a las amplias perspectivas de incorporación de diversidad de estrategias textuales, involucradas en la IC, las libertades del investigador se potencian al rescatar la voz directa de los participantes e, incluso, ser un protagonista más del proceso de investigación (Gergen, Josselson y Freeman, 2015; Packer, 2013; Guba y Lincoln, 2012). De manera específica, el uso de la investigación poética, se relaciona al componente hipertextual propio del tuit como una entidad de sentido independiente, que puede ser conectada con otros tuits y crear diferentes lógicas de sentido. Además, como lo propone Andersen (2017), esta forma de creación se convierte en un tipo de literatura serializada, al incorporar largas secuencias de tuits y re-tuits como manifestaciones de una estructura creativa, más cerca al verso que a la prosa. El uso extendido de esta forma de investigación en ciencias sociales, permite comprender fenómenos como la escritura en red, la escritura compartida, la "twitteratura" y otros ejercicios de escritura en colaboración (Nie, 2015; De Pietri, 2016; Malaver, 2015; Ahmed y Bath, 2015), lo que requiere el uso de metodologías específicas, que den cuenta del potencial narrativo y sensible de estas formas de escritura. 
Para Prendergast (2009), la investigación poética, al ser un estudio sistemático de la poesía como recurso desarrollado en algún componente de la investigación cualitativa, tiene una serie de categorías o tendencias en la distinción de su voz dentro de la investigación: la voz teoría (vox theoria), que consiste en poemas con voz literaria; poemas escritos desde o como una respuesta a trabajos/teorías en una disciplina o campo de conocimiento. La voz de la autobiografía/autoetnografía (vox autobiographia/autoethnographia), que son poemas escritos creados a partir de notas de campo y producto de la escritura reflexiva y creativa del investigador en el desarrollo de una investigación, a modo de un ejercicio autoetnográfico. Finalmente está la voz participante (vox participare), que son poemas escritos a partir de entrevistas transcritas o solicitadas directamente a los participantes de la investigación. Esta voz puede ser singular o múltiple, es decir, solo desde la perspectiva de los participantes o en diálogo de estos con el investigador.

Otros autores, como Gold (2012), al afirmar el potencial del espacio interactivo construido de la poesía relacional propuesta por Witkin (2007), mencionan que la poesía relacional permite la comprensión de nuevas posibilidades de conversación en la práctica de significados sobre la cotidianidad, además que establece una práctica de uso creativo en el que se configuran nuevas subjetividades centradas en el verso, precisamente por su carácter semiótico denso, el cual permite la libertad de interpretación, a la vez que ancla sentidos nuevos, mediados en la conversación y la interacción digital. De acuerdo a los argumentos anteriores, se realizará el abordaje metodológico de la cuenta de Twitter@Elhombredetweed, a partir de estas orientaciones metodológicas, especialmente orientadas a identificar la voz del autor de a cuenta y las redes que construye en la experiencia de intercambio de tuits con otros usuarios.

\section{Población}

La cuenta de Twitter analizada en este artículo es @Elhombredetweed. Esta cuenta fue creada por el escritor mexicano Mauricio Montiel Figueiras. En una entrevista realizada al autor afirma que el interés por crear esta cuenta fue el de:

...generar una narración por entregas que mantenga en vilo al lector... El proyecto del hombre de tweed, que me gusta rubricar como "novela de folletweet", toma como punto de partida la estrategia episódica del folletín para desarrollar un relato de bordes fantásticos que logre continuidad y suspenso mediante párrafos concentrados en ciento cuarenta caracteres (Montiel, s.f.).

Se analizaron, en consecuencia, 1096 tuits y retuits publicados entre el 25 de junio de 2015 al 2 de julio de 2016. Se consideraron no solo los tuits del autor de la cuenta, sino las interacciones de otros usuarios a la cuenta de Montiel.

\section{Instrumentos}

Los tuits y retuits de @Elhombredetweed fueron analizados mediante el software Netlytic (netlytic.org), herramienta de acceso abierto y creada por el Social 
Media Lab, vinculada a la Universidad Ryerson. El análisis de este software estuvo centrado a las interacciones de los usuarios, nubes de palabras y frecuencia de intercambio de tuits entre los usuarios, que retuitearon algún tuit de @ Elhombredetweed.

\section{Procedimiento}

Se realizó un análisis descriptivo de los tuits publicados en el rango de fechas especificado arriba, mediante el software Netlytic, con el fin de establecer un rango de coincidencias en los tuits publicados y retuiteados, de tal manera se pudiesen construir mapas de interacciones entre los usuarios y establecer cuáles tuits fueron más destacados. Asimismo, este software permitió identificar tendencias en los temas publicados e identificar las características de construcción de nodos y clústers construidos en las diferentes interacciones de los usuarios. Además de la interacción con el software, se realizó un análisis categorial de ciertos temas recurrentes en las formas de interacción entre los usuarios de Twitter, con el fin de identificar potencialidades y tendencias en la manera como el autor mexicano ha construido su proyecto literario.

\section{Resultados}

Se describen los resultados encontrados en la cuenta @Elhombredetweed. Para comenzar, se puede afirmar que el movimiento de la cuenta en el rango de tiempo establecido muestra una tendencia al uso del tuit y retuit por parte de@Elhombredetweed, quien de manera reiterada, con un 21.5\% aparece en segundo lugar; la cuenta @ ElGerryChicago, con un $23.7 \%$, es la que lidera el uso de tuits y retuits. La Figura 1 , resume los principales usuarios intervinientes en la producción de información:

Figura 1. Usuarios con mayor frecuencia en producción. Fuente: elaboración propia ${ }^{2}$
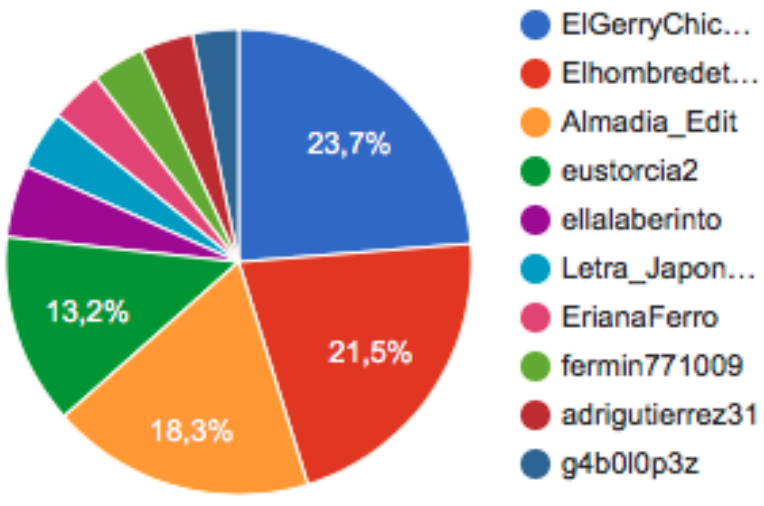

2 La elaboración de los gráficos ha sido realizada con Netlytic: http://Netlytic.org 
En lo relacionado a los clústers, entendidos como una serie de ordenadores interconectados dentro de una red, en la Figura 2, se identifican qué usuarios replicaron a otros usuarios de la red. El color amarillo representa la cuenta @Elhombredetweed, que es la que más replica la producción de otros tuits. Uno de los atributos de la cuenta de Montiel, es la constante réplica de tuits, que se convierte en insumo potencial para el diálogo literario. El objetivo de este proyecto literario, es la creación de una novela por entregas, centrada en la figura de dos protagonistas de ficción el hombre de tweet y la mujer de M. A partir de estos diálogos, la novela evoluciona y se desarrolla en una analogía a las estaciones del año.

Figura 2. Cadena de clústers. De quién replica a usuarios. Fuente: elaboración propia ${ }^{3}$

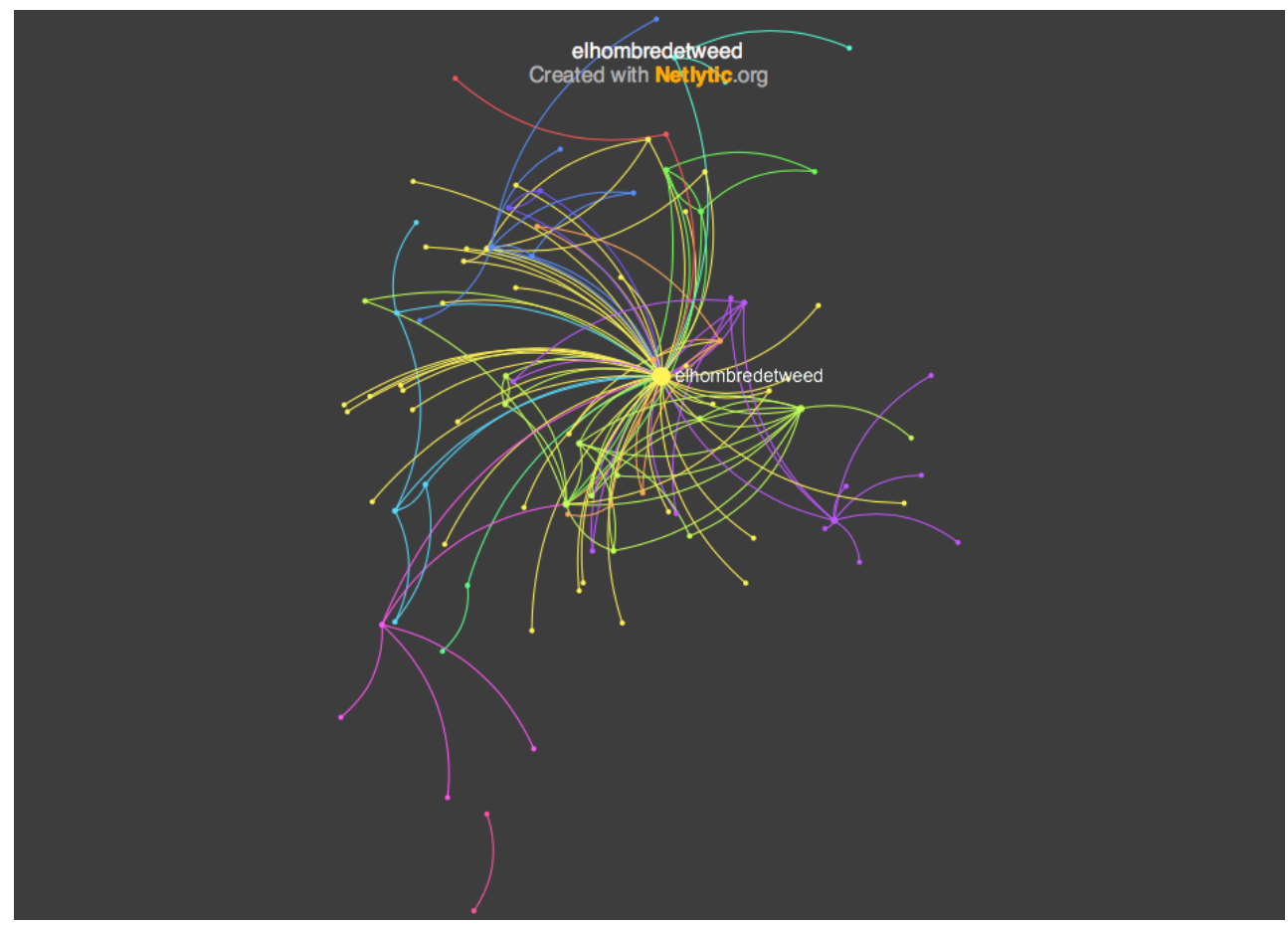

Si se observa con atención, cada color representa a un usuario de la red y en cada uno de los clústers identificados se muestra el número de réplicas que hicieron a otros usuarios. Se entiende aquí la réplica como una respuesta a otro tuit. Para tomar dos ejemplos, en el caso del usuario george_szirtes, ver Figura 3, se identifica una réplica a la cuenta@Elhombredetweed y una serie de réplicas simples a otros siete tuits. Cada usuario aporta una serie de conexiones, o nodos, que permite el tránsito de la información emitida, ampliando el espectro de las interacciones y sus correspondientes respuestas y comentarios.

3 La elaboración de los gráficos ha sido realizada con Netlytic: http://Netlytic.org 
Figura 3. Interacciones de usuario george_szirtes. Fuente: elaboración propia

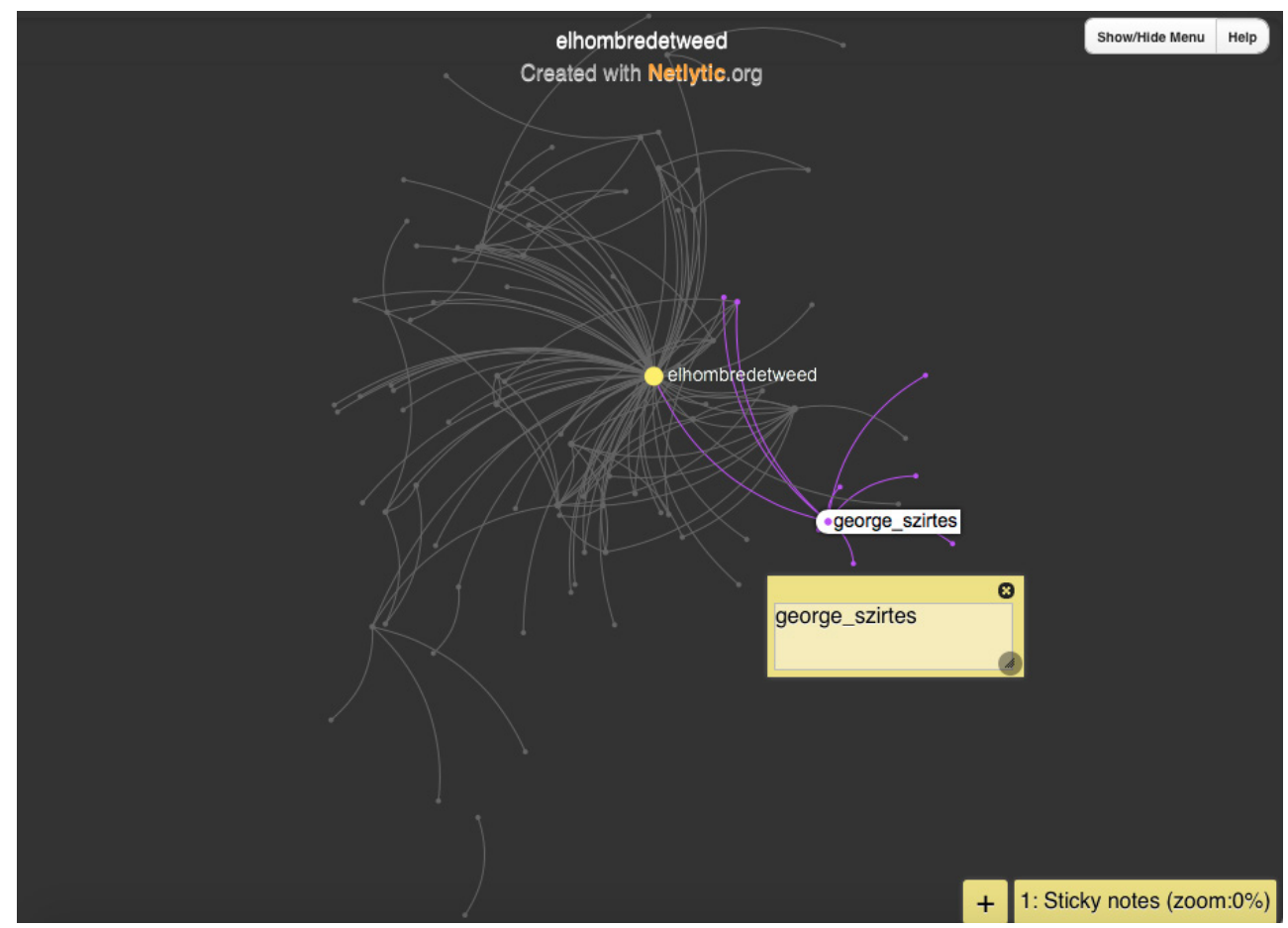

En el caso de las interacciones del usuario moravicenteluis, como se ve en la figura 4 , se crea otro micro nodo, partiendo de una réplica a un tuit de @Elhombredetweed, sumadas a otras cuatro interacciones simples con otros usuarios. 
Figura 4. Interacciones de usuario moravicenteluis. Fuente: elaboración propia

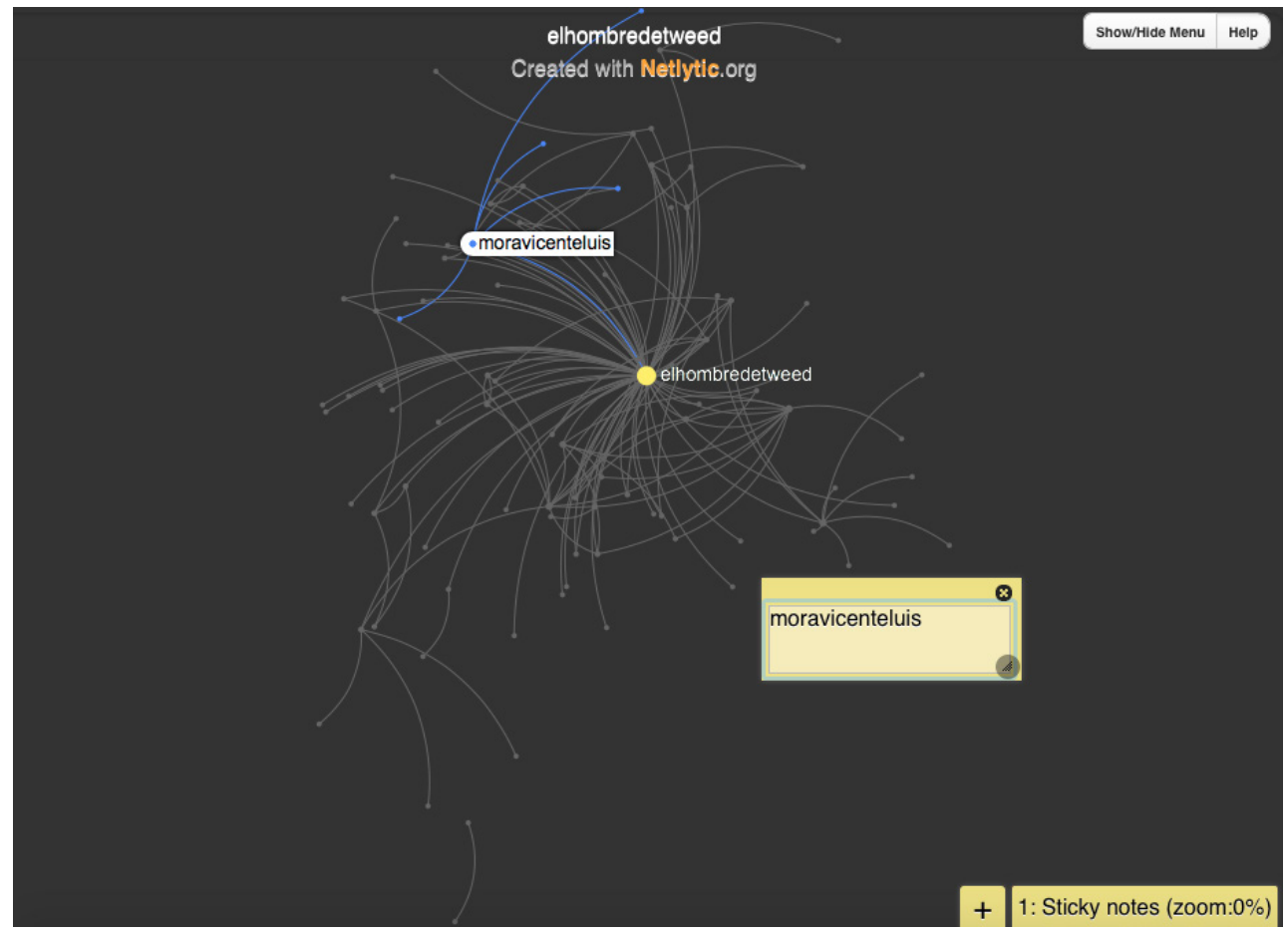

Esto indica que cada nodo (cada usuario) genera su propia constelación de réplicas, en conexión directa con la de @Elhombredetweed, sumada a otras producciones de otros nodos, lo que complejiza el entramado de las interacciones, realzando características centradas bien sea en el contenido de la forma o en la calidad del texto producido. Estas réplicas al ser respuestas directas a un tuit, tienen la condición inicial de ser mencionadas primero (mentions whom) y luego la acción de respuesta al tuit emisor.

En lo referido a quién refiere cuáles usuarios, la constelación de nodos o conexiones se complejiza, dado que en este tipo de interacción se visualiza cada vez que un usuario menciona dentro del mensaje de tuit a otra cuenta. Como se observa en figura 5, se identifican todas las menciones realizadas entre los usuarios, con una fuerte tendencia (desde el centro superior de la imagen) a incluir la cuenta de @Elhombredetweed. Esto refleja el interés por seguir bien sea un tema propuesto desde la cuenta de Montiel o en hacer asociaciones entre usuarios que tienen sus propias redes (más pequeñas) de seguidores. Esto permite que se puedan extender no solo los seguidores de redes más pequeñas, sino potencializa a las cuentas con más seguidores. 
Figura 5. Menciones a otros usuarios en la red. Fuente: elaboración propia

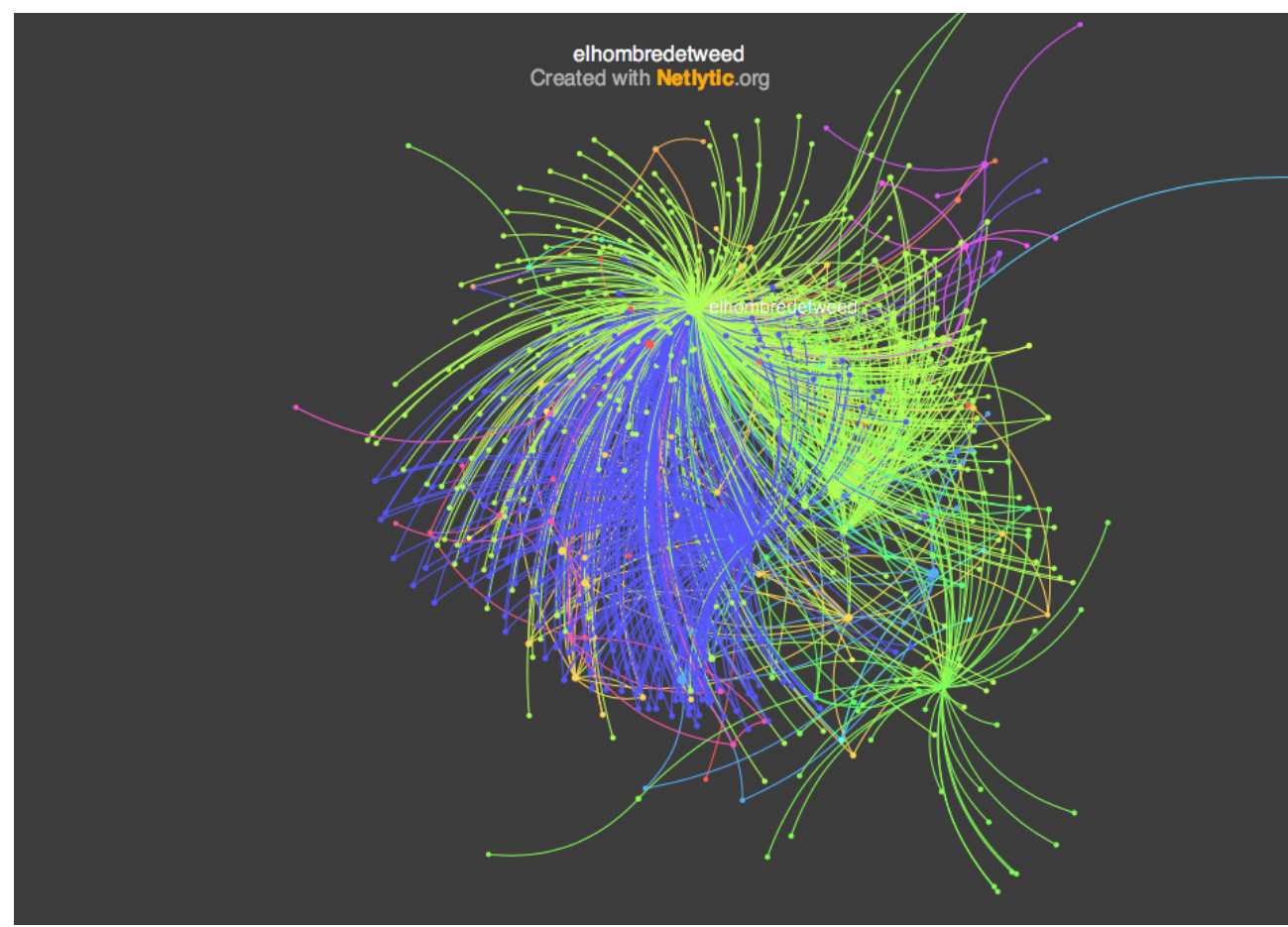

Para poner un ejemplo más concreto, esta es la red de menciones desde el usuario oniropolis (Figura 6), en la que refiere tres tuis a @Elhombredetweed y otra serie de referencias a diferentes usuarios, de una red que se expande de manera significativa, de acuerdo a las interacciones que construye con otros usuarios. Todo este tipo de conexiones están vinculadas a una gradación total (total degree), en el que se combinan las grados de entrada a una cuenta (indegree) y los grados de salida a otra cuenta (outdegree). 
Figura 6. Menciones de usuario oniropolis en la red. Fuente: elaboración propia

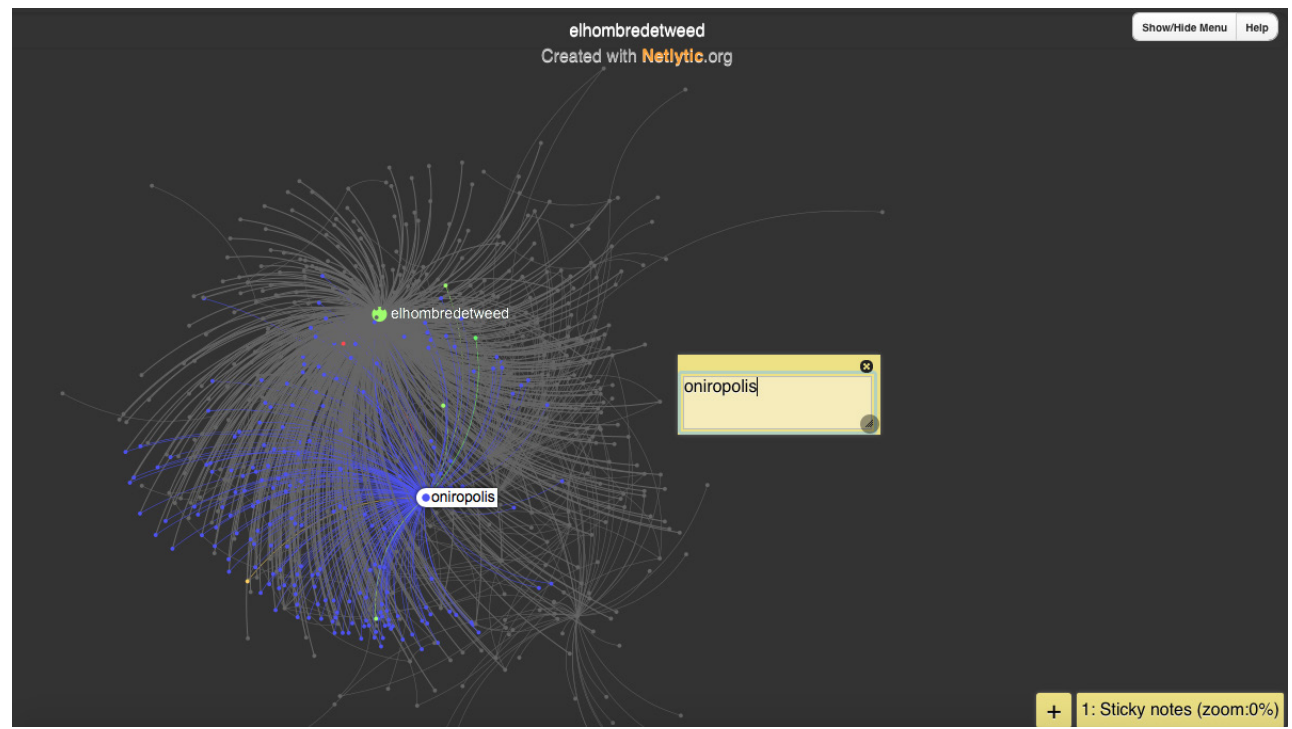

\section{Discusión de resultados}

Uno de los aportes de la psicología cultural es la consideración significativa de los artefactos sociales, si se piensa en la comprensión de los fenómenos humanos vinculados a actividades humanas (Cole, 1999; Esteban y Ratner, 2010; Glaveanu, 2010). Este hecho se debe considerar para el caso del uso de la cuenta@Elhombredetweed, en el que un proyecto literario aborda el sentido de la colaboración literaria, vinculada a la experiencia de uso de los tuits y del valor literario que se les da.

Como se afirma en los resultados, los tipos de interacción, menciones y retuits seguidos por@Elhombredetweed, generan una constelación de historias, vinculadas a una historia de fondo, desconocida para el resto de los usuarios. Esto hace pensar en la tesis de Piglia (2000) sobre las dos historias de un cuento. Para el autor argentino: "Cada una de las dos historias se cuenta de modo distinto" (p. 106). En este sentido no es una historia que requiere ser develada, sino que implica otros procedimientos: "El cuento es un relato que encierra un relato secreto. No se trata de un sentido oculto que depende de la imaginación: el enigma no es otra cosa que una historia que se cuenta de un modo enigmático" (Piglia, 2000, p. 107).

Al igual que el argumento de Piglia con@Elhombredetweed existe la historia, - la intención del autor de la cuenta - y la serie de interacciones que se han creado, a partir de los tuits de los diferentes usuarios. Para retomar la intención del proyecto creativo de la cuenta de Montiel, se pueden identificar estas características del proceso de creación. En primer lugar, sobre el cómo surgieron los personajes de las cuentas de Twitter, ante lo cual responde Montiel: "El hombre de tweed y la mujer de M. (@LamujerdeM), los personajes centrales del proyecto narrativo que desarrollo en Twitter desde 2011, surgieron de la observación de esos seres solitarios que suelen pasar inadvertidos en medio del bullicio cotidiano" (Montiel, s.f., p. 1).

En segundo lugar, dado que se han creado las condiciones narrativas de una suerte de personajes, diferentes a la figura del yo de carne y hueso del autor, Montiel establece unas diferencias frente a la producción de los tuits. Afirma el autor: 
Así pues, los tweets entre corchetes me corresponden a mí, Mauricio Montiel, mientras que los que carecen de corchetes son parte de la narración del hombre de tweed. ¿Complicado? Más bien divertido: las nuevas plataformas de escritura como Twitter también se prestan para poner en práctica el espíritu lúdico (Montiel, s.f., p. 2).

En tercer lugar, al identificarse las voces de los protagonistas de la narración y las del propio autor, se establece una ruta prevista para la narración de la obra ciberliteraria, la cual explica de esta forma el autor mexicano:

El proyecto novelístico protagonizado por el hombre de tweed consta al día de hoy de tres partes; cada una corresponde a una estación del año (primavera, verano, otoño) y a una geografía específica (una ciudad, una isla, una Venecia en la que confluyen distintas épocas históricas). Queda pendiente por escribir la cuarta y última parte del proyecto, correspondiente al invierno, que se desarrollará en el pueblo fantasma donde vive la mujer de M. (Montiel, s.f., p. 2).

De este modo, la historia secreta, por decirlo así, o la historia central de esta novela en folletín de Montiel, se estructura alrededor de una idea preconcebida del autor, a la que poco a poco, de manera sistemática, con o sin intención, pero centradas en el interés por la creación y la colaboración en red, se suma una serie de intervenciones por parte de los usuarios de Twitter alrededor de la experiencia de uso de esta red social.

Resulta importante mencionar que, en consecuencia, una serie de voces se manifiestan en una sola cuenta de Twitter, a la vez que se rehace la experiencia de lectura y reconstrucción de quienes siguen la cuenta y que, probablemente, no conocen la historia de fondo, el contexto literario que persigue@Elhombredetweed. En consecuencia, se cruzan destinos literarios, se esbozan proyectos de escritura cortos, breves, encapsulados en momentos de interacción, a la vez que se mantiene y expande el proyecto de escritura general.

Sin duda alguna el análisis de las cuentas de Twitter y la discusión cultural de la cognición en tiempos de la postmodernidad, sus usos mentales online y la importancia de la colaboración como estrategia de producción artística, se configuran como ejes comprensivos de formas emergentes de literatura que permiten pensar en la posibilidad de nuevos cánones para su estudio y apropiación por parte de la comunidad. Esta diversidad de voces se configura como momentos de construcción subjetiva, más allá de las relaciones cara a cara, lo que impulsa el sentido construccionista social, esencial, de un yo relacional que se consolida en la relación instantánea del mundo digital (Gergen y Gergen, 2011; Gergen, 2016). En este sentido, se considera el siguiente argumento de Krieger cuando afirma de la necesidad de pensar epistemologías que incluyan la variedad de sensaciones y percepciones que puede proporcionar la experiencia digital: "Una epistemología de la percepción multisensorial que oscila con acentos desiguales entre racionalidades cognitivas, emotivas y corporales co-presentes y entre experiencia estética y vivencia cotidiana" (Krieger, 2012, p. 188).

Lo anterior indica que la experiencia de lectura y escritura de Twitter es la a vez el reconocimiento de una epistemología que no solo debe centrarse en el aspecto intrínseco de una producción literaria particular, extraña o ajena al mundo de las 
grandes ideas académicas, sino que es un asunto de las lógicas particulares de las personas, en el entorno de sus mundos cotidianos. También configura otra forma de identidad, como la propuesta de Elwell (2014), centrada en lo integrado, lo disperso, lo episódico y lo interactivo.

Finalmente, el aporte de este tipo de ejercicios literarios colaborativos a las humanidades digitales, confirma el potencial que tiene la construcción de la subjetividad online, como un campo de estudio que merece ser estudiado. Como afirman Wagner-Pacifici et al. (2015) las construcciones de la categoría individuo, más que nunca deben reconocer las características a las que el mundo digital invita con experiencias de lectura de la realidad, en términos de dualidades que se complementan, como es el caso de la relación mente/máquina, inducción/deducción o vida/datos. La revolución de los datos, el carácter empírico y las transformaciones de la manera como se realizan las prácticas de investigación (Kitchin, 2014), requiere de nuevas aproximaciones y, en este caso, la experiencia colaborativa de construcción de textos literarios, como es el caso de@Elhombredetweed, es una claro ejemplo, entre otra cantidad increíble de aproximaciones teóricas y literarias, del potencial de abordaje de la experiencia humana, mediada por las tecnologías digitales.

\section{Conclusiones}

Se puede concluir que la experiencia literaria de @Elhombredetweed se acerca a la propuesta transmedia de Elwell (2014) cuando se identifican los cuatro momentos de enunciación del acontecimiento transmediado (lo integrado, lo disperso, lo episódico y lo interactivo). En primer lugar, existe el yo integrado que es el sentido intencional de la cuenta de@Elhombredetweed al configurar una experiencia literaria, en el lugar de enunciación de la trama general de la novela que propone Montiel, además de la estructura de la obra misma, en la que se pretende establecer un texto completo, un texto tipo novela, expresada en entregas cortas, como es el caso del uso del tuit como expresión mínima de este ejercicio literario. Esto se puede pensar como una expansión/ exploración digital de la literatura folletinesca de finales del siglo XIX y comienzos del siglo XX.

En segundo lugar, el yo disperso online de los tuits se presenta en todo el espectro de uso, apropiación y expansión de las historias contadas en 140 caracteres, sin que haya un control o una indicación a un orden predeterminado por parte del escritor mexicano. Al recorrerse la cuenta se pueden identificar distintos niveles de uso de los tuits que se alternan entre la voz propia del autor, la voz de los personajes del proyecto literario y las expresiones y respuestas por parte de los distintos usuarios que tienen interacción con la cuenta de Montiel. En tercer lugar, el yo episódico de la constelación de tuits permite cierta identificación de momentos creativos, en los que se crean las condiciones para la creación de historias, o el uso de algún tuit con el fin de provocar la reacción específica de los usuarios, sin que haya inicio o fin de la instrucción. En cuarto lugar, la interactividad se hace palpable por dos mecanismos: la mención de la cuenta de entrada o de salida al tuit de interés y la réplica específica, la respuesta al tuit como motivador para contar microescrituras entre usuarios previamente identificados.

Finalmente, esta construcción colectiva de historias, vinculadas a la cuenta@Elhombredetweed, permite pensar los indiscutibles usos para la investigación poética en ciencias sociales, ya que permite comprender que en el uso de las historias cortas, 
se crean significados específicos sobre realidades concretas de la vida humana, bien sea contadas a través de mecanismos ficcionales y autoficcionales, así como reacciones a tuits como la expresión de afectos y sentimientos evocadores. Esto sin duda abre el camino a la construcción comunal y colaborativa de procesos psicológicos, ajenos a una modernidad centrada en la figura exclusiva de un autor o un intérprete único y centralizado. En otras palabras, se construye un yo mediado por las transacciones electrónicas, que lo actualizan y potencian en la red.

\section{Referencias}

Ahmed, W. y Bath, P. A. (2015). Comparison of Twitter APIs and tolos for analysing Tweets related to the Ebola Virus Disease. In: iFutures 2015. iFutures: iSchool Postgraduate Research Conference, 7 July 2015, [en línea]. Disponible en http://eprints.whiterose. ac.uk/87868/

Andersen, T. (2017). Straggered transmissions: Twitter and the return of the serialized literature. Convergence: The International Journal of Research into New Media Technologies, 23(1), 34-48. DOI: 10.1177/1354856516675256

Begoña, S. (2012). ¿Qué es poesía?: La literariedad en la poesía digital. En R. Ferrer y F. Chico (Eds). Ciberliteratura y comparitivismo (pp. 233-247). Alicante: Universidad de Alicante y Sociedad Española de Literatura General y Comparada.

Bullingham, L. y Vasconcelos, A. (2013). 'The presentation of self in the online world': Goffman and the study of online identities. Journal of Information Science, 39(1), 101112. Doi: $10.1177 / 0165551512470051$

Chico, F. (2007). Literatura y teoría literaria en la era digital. En M. Utrera y M. Romero (Eds). Estudios literarios in honorem Esteban Torre (pp. 787-800). Sevilla: Universidad de Sevilla.

Clark, W., Couldry, N., MacDonald, R. y Stephansen, H. (2015). Digital platforms and narrative Exchange: Hidden constraints, emerging agency. New media y society, 17(6), 919-938. Doi: 10.1177/1461444813518579

Cole, M. (1999). Psicología cultural. Madrid: Morata.

Cook, J.yHasmath, R.(2014). The discursive construction and performance of gendered identity on social media. Current sociology, 62(7), 975-993. Doi: 10.1177/0011392114550008

Couldry, N., MacDonald, R., Stephansen, H., Clark, W., Dickens, L. y Fotopoulou, A. (2015). Constructing a digital storycicle: Digital infrastructure and mutual recognition. International Journal of Cultural Studies, 18(5), 501-517. Doi: 10.1177/1367877913519313

Cravero, G. (2011). Hiperliteratura: nuevas experiencia de escritura en formato weblog. Revista Austral de Ciencias Sociales, 20, 61-69, [en línea]. Disponible en http:// mingaonline.uach.cl/pdf/racs/n20/art04.pdf

Deegan, M. (2014). 'This ever more amorphous thing called Digital Humanities': Whiter the Humanities Project? Arts \& Humanities in Higher Education. 13(1-2), 24-41. Doi: $10.1177 / 1474022213513180$

De Pietri, V. (2016). Wago WagōRyōichi's Net-poetry and the revolucionary 'Shared Literature'. Annali di $\mathrm{Ca}^{\prime}$ Foscari. Serie orientale, 52, giugno. 351-370. Doi: 10.14277/2385-3042/ AnnOr-52-16-13

Doh, Y. y Whang, S-M. (2014). From Separation to Integration: Identity Development of Korean Adult Players in Online Game World. Games and Culture, 9(1), 30-57. Doi: $10.1177 / 1555412013498301$ 
Elwell, J. (2014). The transmediated self: Life between the digital and the analog. Convergence: The international Journal of Research into New Media Technologies, 20(2), 233-249. Doi: 10.1177/1354856513501423

Esteban, M. y Ratner, C. (2010). Historia, conceptos fundacionales y perspectivas contemporáneas en psicología cultural. Revista de historia de la psicología, 31(2-3), 117 136.

Felt, M. (2016). Social media and the social sciences: How researchers employ big data analytics. Big data y society, 3(1), 1-16. Doi: 10.1177/2053951716645828

Floridi, L. (2011). The Construction of Personal Identities Online. Minds y Machines, 21, 477-479. Doi: 10.1007/s11023-011-9254-y

Gal, N., Shifman, L. y Kampf, Z. (2016). "It Gets Better": Internet memes and the construction of collective identity. New media y society, 18(8), 1698-1714. Doi: $10.1177 / 1461444814568784$

Gergen, K. (2016). Toward a Visionary Psychology. The Humanistic Psychologist, 44(1), 3-17. Doi: 10.1037/hum0000013

Gergen, K., Josselson, R. y Freeman, M. (2015). The promises of qualitative inquiry. American psychologist, 70(1), 1-9. Doi: 10.1037/a0038597

Gergen, K. y Gergen, M. (2011). Reflexiones sobre la construcción social. España: Paidós.

Gergen, K. (2006). El yo saturado. Dilemas de identidad en el mundo contemporáneo. España: Paidos.

Gergen, K. (1998). From control to coconstruction: new narratives for the social sciences. Psychological Inquiry, 9(2), 101-103, [en línea]. Disponible en http://www.jstor.org/ stable/1449100.

Glaveanu, V. (2010). Principles for a Cultural Psychology of Creativity, Culture psychology, 16(2), 147-163. Doi: 10.1177/1354067X10361394.

Gold, K. (2012). A space for stories: revisting relational poetry as inquiry on everyday practice. Qulitative social work, 12(6), 849-857. Doi: 10.1177/1473325012464805

Guba, E. y Lincoln, Y. (2012). Controversias paradigmáticas, contradicciones y confluencias emergentes. Desafíos y perfiles. En N. Denzin y Y. Lincoln (Coords). Manual de Investigación cualitativa. Vol. II. Paradigmas y perspectivas en disputa (pp. 38-78). España: Gedisa.

Jenkins, H. (2010). Transmedia Storytelling and Entertainment: An annotated syllabus, Continuum: Journal of Media y Cultural Studies, 24(6), 943-958. Doi: 10.1080/10304312.2010.510599

Jenkins, H. (2008). Convergence Culture. Barcelona: Paidós.

Jenkins, H. (2004). The cultural logic of media convergence. International Journal of cultural studies, 7(1), 33-43. Doi: 10.1177/1367877904040603

Jenkins, H. y Deuze, M. (2008). Editorial. Convergence culture, Convergence, 12(1), 5-12. Doi: $10.1177 / 1354856507084415$

Karhio, A. (2017, Junio 3). The end of Landscape: Holes by Graham Allen. Electronic book review, . [en línea]. Disponible en http://electronicbookreview.com/thread/electropoetics/ landscaped

Kateros, S., Georgiou, S., Papaefthymiou, M., Papagiannakis, G. y Tsioumas, M. (2015). A comparison of Gamified, immersive VR Curation Methods for Enhanced Presence and Human-computer Interaction in Digital Humanities. International Journal of Heritage in the digital Era, 4(2), 221-233. Doi: 10.1260/2047-4970.4.2.221

Kitchin, R. (2014). Big data, new epistemologies and paradigm shifts. Big Data \& Society, april-june, 1-12. Doi: 10.1177/2053951714528481 
Kozak, C. (2015). Literatura digital y materialidad. Cómo se lee. Artnodes, 15, 90-98, [en línea]. Disponible en http://artnodes.uoc.edu

Krieger, H. (2012). Literatura digital: una nueva relación entre teoría y práctica experimental. En R. Ferrer y F. Chico (Eds). Ciberliteratura y comparitivismo (pp. 181-190). Alicante: Universidad de Alicante y Sociedad Española de Literatura General y Comparada.

Landow, G. (1995). Hipertexto. La convergencia de la teoría crítica contemporánea y la tecnología. España: Paidós.

Landow, George (1997). Teoría del hipertexto. España: Paidós.

Levy, P. (2007). Cibercultura. Informe al consejo de Europa. España: Anthropos.

Levy, P. (2004). Inteligencia colectiva. Washington: OPS.

Malaver, A. (2015). Microrrelato y micrometraje: Paradigmas contemporáneos de la brevedad en la literatura y el cine latinoamericano y español. Aproximaciones teóricas y éticas, [en línea]. Disponible en https://getd.libs.uga.edu/pdfs/malaver-copara_ary_e_201505_phd.pdf

Montiel, M. (s.f.). Comunidades ciberliterarias: Una lectura comprensiva a partir de la psicología cultural. Entrevista de opinión [documento de word].

Nagy, P. y Koles, B. (2014). The digital transformation of human identity: Towards a conceptual model of virtual identity in virtual worlds. Convergence: The international journal of research into new media technologies, 20(3), 276-292. Doi: $10.1177 / 1354856514531532$

Nesteruk, J. (2015). Digital Storytelling: Bringing Humanistic Inquiry to Management Studies. Journal of Mangement Education, 39(1), 141-152. Doi: 10.1177/1052562914545335

Neves, C. (2010). Da poesía visual concreta à poesía virtual concreta: a ciberliteratura na sala de aula. Educaçao Temática digital, 12(1), 124.146, [en línea]. Disponible en http:// nbn-resolving.de/urn:nbn:de:0168-ssoar-212251

Nie, B. (2015). Exploring Image Culture Through Narrative: A Study on Jennifer Egan's Twitter Fiction Black Box. Journal of Literature and Art Studies, 5(10), 820-829. Doi: 10.17265/2159-5836/2015.10.002

Packer, M. (2013). La ciencia de la investigación cualitativa. Bogotá: Universidad de los Andes.

Piglia, R. (2000). Formas breves. Barcelona: Anagrama.

Prendergast, M. (2009). Introduction: The Phenomena of Poetry in Research: "Poem mis What? Poetic Inquiry in Qualitative Social Science Research. In M. Prendergast, C. Leggo y P. Sameshima (eds). Poetic Inquiry. Vibrant Voices en the Social Sciences (pp. xix-xlii). Rotterdam: Sense Publishers.

Quan-Hasse, A., Martin, K. y McCay-Peet, L. (2015). Networks of digital humanities scholars: The informational and social uses and gratifications of Twitter. Big data \& society, 2(1), 1-12. Doi: 10.1177/2053951715589417

Quan-Hasse, A., Suarez, J. y Brown, D. (2015). Collaborating, Connecting, and Clustering in the Humanities: A Case Study of Networked Scholarship in an Interdisciplinary Dispersed Team. American Behavioral Scientist, 59(5), 565-581. Doi: 10.1177/0002764214556806

Radovanović, D. Hogan, B. y Lalić, D. (2015). Overcoming digital divides in higher education: Digital literacy beyond Facebook. New media \& society, 17(10), 1733-1749. Doi: $10.1177 / 1461444815588323$

Romero, D. (2011). La literatura digital en español: Estado de la cuestión. Texto digital, 7(1), 38-66. Doi: 10.5007/1807-9288.2011v7n1p38

Scolari, C. (2013a). Media Evolution: Emergence, Dominance, Survival and Extinction in the Media Ecology. International Journal Of Communication, 7(24), 1418-1441, [en línea]. Disponible en http://ijoc.org/index.php/ijoc/article/view/1919/936 
Scolari, C. (2013b). Lostology: Transmedia storytelling and expansion/compression strategies. Semiotica, 195, 45-68. Doi 10.1515/sem.2013.0038

Scolari, C. (2013c). Narrativas transmedia. Cuando todos los medios cuentan. Barcelona: Planeta.

Simanowski, R. y Gattass, L. (2017, Marzo 5). Debates in the Digital Humanities formerly known as Humanities Computing. Electronic book review, [en línea]. Disponible en http://electronicbookreview.com/thread/electropoetics/debated

Szilak, I. (2015, Agosto 2). Towadrs Minor Literature Forms: Digital Literatura and the Art of Failure. Electronic book review, [en línea]. Disponible en http://electronicbookreview. $\mathrm{com} /$ thread/electropoetics/failure

Tsatsou, P. (2017). Literacy and training in digital research: Researchers' views in five social science and humanities disciplines. New media y Society, 1-20. Doi: $10.177 / 14614481668274$

Valls-Russell, J. (2016). Digital Shakespeare: An expanding universo in search of itself. Cahiers Élizabéthains: A Journal of English Renaissance Studies, 91(1), 82-87. Doi: $10.1177 / 0184767816663126$

Vilariño, T. (2013). Tecnologías literarias: La oralidad en la poesía digital. Pasavento. Revista de estudios hispánicos. 1(2), 217-229.

Wagner-Pacifici, R., Mohr, J. y Breiger, R. (2015). Ontologies, methodologies, and new uses of Big Data in the social and cultural studies. Big Data \& Society, july-december, 1-11. Doi: $10.1177 / 2053951715613810$

Wessels, B. (2012). Identification and the practices of identity and privacy in everyday digital communication. Newmedia y society, 14(8), 1251-1268. Doi: 10.1177/1461444812450679

Witkin, S. (2007). Relational poetry: Expressing interweaving realities. Qualitative Social Work, 6(4): 477-481. Doi: 10.1177/1473325007083358

Wittig, R. (2015, Noviembre 1). Pasts and futures of Netprov. Electronic book review, [en línea]. Disponible en http://electronicbookreview.com/thread/electropoetics/netprov

Zambrano, F. (2015). La creación literaria en 140 caracteres. En Dossier. Escritura analógica y escritura digital. Boletín de la Academia Venezolana de la Lengua, [en línea]. Disponible en https://avelengua.org.ve/cms/dossier-escritura-analogica-y-escritura-digital/ 